\title{
A DNA Vaccine Against Infectious Hematopoietic Necrosis Virus
}

\author{
SCOTT E. LAPATRA ${ }^{1}$, NIELS LORENZEN ${ }^{2}$, AND GAEL KURATH ${ }^{3}$
}

${ }^{I}$ Clear Springs Foods, Inc., Research Division, P.O. Box 712, Buhl, Idaho, 83316, USA (scottl@clearsprings.com); ${ }^{2}$ Danish Veterinary Laboratory, Hangovej 2, DK-8200 Aarhus N, Denmark; and USGS Western Fisheries Research Ceneter, 6505 NE $65^{\text {th }}$ st., Seattle, Washington 98115, USA

SUMMARY: A DNA vaccine against infectious hematopoietic necrosis virus (IHNV) was the first DNA vaccine developed for use in salmonid fish. Protective immunogenicity was only observed with the glycoprotein (G) gene in rainbow trout Oncorhynchus mykiss using the DNA vaccine technology. A similar significant level of protection was also observed in Atlantic salmon Salmo salar vaccinated with a naked plasmid encoding the $G$ protein and assessed for protection by immersion and cohabitation IHNV challenges. In both studies vaccinated fish seroconverted and this sera was shown to be protective after passive transfer and subsequent challenge with IHNV. Vaccine doses as low as 1-10 ng provided significant protection to rainbow trout fry (mean weight, 0.8-1.8 g) and an optimal dose of $0.1 \mu \mathrm{g}$ was selected. Rainbow trout immunized with this dose were shown to significantly protected against a broad range of viral strains from different geographic locations and host species. Although intramuscular injection is the principle mode of vaccination, gene gun immunization induced protective immunity in fry, while intraperitoneal injection provided partial protection. This vaccine was also shown to provide significant protection as soon as 4 days after intramuscular vaccination and provided a significant level of crossprotection against another fish rhabdovirus, viral hemorrhagic septicemia virus, for a transient period of time.

KEY WORDS: IHNV, infectious hematopoietic necrosis virus, fish vaccine, DNA vaccine

\section{INTRODUCTION}

The most significant thabdoviral disease of salmon and trout in North America is infectious hematopoietic necrosis (IHN). Annual loses of several millions of dollars occur among commercially-reared rainbow trout Oncorhynchus mykiss and IHN also affects wild and cultured Pacific salmon, steelhead O. mykiss, and Atlantic salmon Salmo salar throughout the Pacific Northwest. The history of IHN vinus (IHNV), its chemical, physical, and serological characteristics, and factors affecting virulence of the virus for salmon and trout have been comprehensively reviewed. ${ }^{1,2)}$

A DNA vaccine delivers to the host the gene for an antigenic protein rather than delivering the protein itself. In many cases this results in a better immune response than traditional vaccines, presumably because the antigenic protein is synthesized in the host in situ in a manner that more closely mimics its synthesis during a natural infection. For fish, the first application of DNA vaccine technology was reported by Anderson et $\left.a\right|^{3)}$ who used a plasmid containing the glycoprotein (G) gene of IHNV to stimulate a protective immune response in rainbow trout fry. The plasmid DNA was injected into the skeletal muscle of rainbow trout fry and immunization was determined by the detection of virus neutralizing antibody activity and by protection against live virus challenge. These studies showed that a DNA vaccine will protect rainbow trout against the lethal effects of IHNV infection. This review will describe further work in our laboratories that suggests that DNA vaccines against IHNV have great potential both as a practical biologic for protection of fish and as important tool for investigation of the teleost immune system.

\section{IMMUNOGENICITY OF OTHER GENES OFIHNV}

The nucleoprotein $(N)$, phosphoprotein $(P)$, matrix protein (M), non-virion protein (NV) and glycoprotein (G) genes of a 1982 IHNV isolate (the WRAC strain) were subcloned downstream of the immediate-early enhancer-promoter sequences of human cytomegalovirus (CMV) of the PCDNA 3.1 
vector. All constructs including a $\beta$-galactosidase construct (pCDNA- $\beta$-gal) were multiplied in Escherichia coli strain DH5 cells grown in Luria broth media, and plasmid DNA was purified with anion-exchange chromatography columns (Quiagen, Chastworth, CA). The capacity of the plasmids to express the $G, N$ and $\beta$-gal proteins in vitro was assessed in transfected EPC cells. The expression of the $\mathrm{G}$ and $\mathrm{N}$ genes was confirmed by indirect immunofluorescence while the $\beta$ galactosidase activity was assessed by incubating transfected cells with $\mathrm{X}$-gal solution at $37^{\circ} \mathrm{C}$ for 16 h.

Rainbow trout fry immunized with each vaccine construct were tested for protection against an IHNV immersion challenge at 4 to 6 weeks postimmunization. In 3 consecutive experiments, rainbow trout fry immunized with 10,5 or $1 \mu \mathrm{g}$ of pCDNA-G exhibited significantly $(p<0.01)$ less mortality than fry vaccinated with the vector alone or with $p C D N A-\beta-g a l$. Cumulative percent mortality in fish immunized with $P C D N A-G$ ranged from 0 to $2 \%$ whereas mortality in fish immunized with DNA vaccines that contained other proteins or were left unhandled ranged form 36 to $76 \%$. There was no significant difference in mortality or mean day to death for fish immunized with pCDNA-N, pCDNA$M$, pCDNA-P or pCDNA-NV relative to control vaccine groups. ${ }^{4}$ ) This confirms the previous report of protection induced by an IHNV G DNA vaccine in rainbow trout fry. These results also suggest that no specific or non-specific immune response(s) were induced by any other IHNV gene in a DNA vaccine construct.

\section{IMMUNOGENICITY OF THE IHNV DNA VACCINE IN OTHER SPECIES}

A naked plasmid DNA encoding the $G$ gene (pCMV4-G) of a 1976 isolate was used to vaccinate Atlantic salmon Salmo salar against IHNV. Eight weeks post-vaccination the fish were challenged with a strain of IHNV originally isolated from farmed Atlantic salmon that had undergone a recent epizootic. Fish injected with the glycoprotein-encoding plasmid were significantly $(p<0.05)$ protected against IHNV by both immersion and cohabitation challenge. Survivors of the first challenge were pooled and rechallenged by immersion 12 weeks after the initial challenge. Significant $(p<0.05)$ protection was observed in all of the previously challenged groups including those receiving the complete vaccine. Fish injected with the glycoprotein-encoding plasmid produced low levels of virus-neutralizing antibodies prior to the first challenge. Neutralizing antibodies increased in all groups after exposure to the IHNV. ${ }^{5}$ )

Sockeye salmon Oncorhynchus nerka (mean weight $150 \mathrm{~g}$ ) were injected with $25 \mu \mathrm{g}$ of the same DNA vaccine and held at a temperature of $12^{\circ} \mathrm{C}$. Six weeks after vaccination, the fish were bled, sera were collected and neutralizing antibody titers were determined as previously described. ${ }^{6}$ ) IHNV neutralization titers ranging from 80 to 320 were detected indicating that these 5 fish responded specifically to IHNV. None of the 4 control fish vaccinated with pCDNA- $\beta$-gal had detectable neutralization titers. Rainbow trout fry were passively immunized with sera obtained from 150 9 sockeye salmon that had been previously immunized with pCDNA-G or pCDNA- $\beta$-gal. Control fry were injected with buffer. Following immersion challenge with IHNV, fry immunized with pCDNA-G and pCDNA- $\beta$-gal had relative survivals of 100 and $58 \%$, respectively, compared to control fry. ${ }^{4)}$

These studies demonstrate that a single intramuscular injection of Atlantic salmon or sockeye salmon with $25 \mu \mathrm{g}$ of DNA vaccine PCMV-G results in significant protection against an IHNV challenge and a high degree of seroconversion. The results extend the findings of earlier studies and demonstrate that viral DNA vaccines are effective in larger size fish (70 to 150 g) and that Atlantic salmon and sockeye salmon can be included in the species that elicit a protective immune response following injection with a DNA vaccine.

\section{MINIMAL VACCINE DOSE IN DIFFERENT SIZES OF RAINBOW TROUT}

Evaluation of the minimal dose of vaccine required for protection was used as an assessment of the potential of the vaccine as a control pharmaceutical. For these studies the plasmid was designated plHNw-G (previously pCDNA-G). The luciferase gene construct ( $p L u c$ ) was made by subcloning the luciferase gene from the PGL3 vector into the pCDNA 3.1 vector. All constructs were amplified in Echerichia coli strain DH5 c cells grown in Luria broth, and plasmid DNA was purified for use as a vaccine following the alkaline 
lysis protocol of Saporito-Irwin et al.

Results showed that rainbow trout fry (average weight $1.8 \mathrm{~g}$ ) vaccinated with $5.0,1.0,0.1$, or 0.01 $\mu g$ of the plHNw-G DNA vaccine exhibited significantly $(p<0.05)$ less mortality than fry vaccinated with the pluc control vaccine or with PBS. Although the minimum effective dose of vaccine was as low as $1-10 \mathrm{ng}, 0.1 \mu \mathrm{g}$ was selected as the optimal dose of plHNw-G in 1-2 g rainbow trout fry to assure consistent strong protection under various conditions. This dose was used as the optimal dose in all future studies of this vaccine in fry. In addition, it was determined that it would cost only 1 cent for reagents to produce a sufficient amount of the plHNw-G plasmid to vaccinate 50 fry at the $0.1 \mu \mathrm{g}$ dose, making the vaccine cost effective to use in commercial aquaculture pending development of methods for mass immunization. ${ }^{8)}$

Another dose-response study that used the plHNw-G vaccine showed that complete and highly significant $(p<0.001)$ protection against a virus injection challenge could be attained in subyearling rainbow trout $(145-160 \mathrm{~g}, 8-$ to $10-$ months-old) 6 weeks after a single intramuscular injection with doses as low as $1 \mu \mathrm{g}$. Complete protection was also reproducibly demonstrated at higher vaccine doses, however, no protection was observed with a $0.1 \mu \mathrm{g}$ dose. Virus-neutralizing antibody titers were detected in fish that had been vaccinated with different doses of the DNA vaccine and then sham-infected. There appeared to be a dose-dependent effect, with higher titers obtained with higher doses of vaccine. The DNA-vaccinated animals that survived virus challenge had significantly $(p<0.05)$ higher neutralizing antibody titers than sham-infected, DNA-vaccinated control fish. Additionally, the titers detected in the IHN survivors exhibited a significant $(p<0.05)$ dosedependent effect, with the highest titers being present in fish that received the highest vaccine doses. ${ }^{9)}$

From an IHN management standpoint, these results suggested that this vaccine may be costeffective where injection vaccination is feasible, such as in valuable stocks of threatened and endangered salmonids and Atlantic salmon that are grown to $2-4 \mathrm{~kg}$ commercially. The $1 \mathrm{\mu g}$ dose of vaccine for a larger fish would cost about $\$ 0.002$ cent. Although significant protection can be obtained with doses as low as $1 \mu \mathrm{g}$ of plHNw-G in sub-yearling rainbow trout, higher vaccine doses may be beneficial because they induce higher antibody titers for long-duration protection against reinfection with IHNV. These results further illustrate the potency of the DNA vaccine against IHNV in rainbow trout and that a single dose of this DNA vaccine can induce protection in fish at a lower dose than is usually reported in mammalian DNA vaccine studies.

\section{PROTECTION AGAINST HETEROLOGOUS STRAINS OF IHNV}

Protection against heterologous strains of IHNV was investigated by vaccinating fry with $0.1 \mu \mathrm{g}$ of plHNw-G or pLuc and challenging them with the homologous WRAC strain and six heterologous strains selected to represent the geographic range and genetic diversity of IHNV. Among the five strains from North America, the WRAC, RB-1, and Col-85 strains were from Idaho, Oregon, and California, respectively, and represented the main phylogenetic lineages of IHNV that have been described. An Alaskan isolate, AK-14, was included to represent the northern portion of the range of IHNV. The 220-90 strain from Idaho was included as a representative of the highly virulent isolates affecting the rainbow trout industry. The Shizuoka strain isolated from rainbow trout in Japan in 1982 and the 32-87 strain isolated from rainbow trout in northern France in 1987 were included as representatives of Asia and Europe, respectively. Six weeks post-immunization rainbow trout fry were tested for protection against the seven IHNV strains by immersion challenge using the highest viral titers obtainable for each strain. Cumulative mortality results showed that fish immunized with the plHNw-G vaccine and challenged with the WRAC, 220-90, AK-14, RB-1, Shizuoka, and 32-87, strains of IHNV were significantly $(p<0.05)$ protected relative to pLuc mock vaccinated fish, showing that the pIHNw-G treatment conferred broad protection. ${ }^{8)}$

The well documented strain diversity of IHNV has prompted recognition that an IHNV vaccine would need to be effective against heterologous viral strains. Previous studies of crossneutralization and cross-protection in vivo have shown that, despite the diversity of IHNV strains, only one serotype of IHNV is identified by polyclonal rabbit antisera, and that the glycoprotein of one IHNV type induces protective immunity against heterologous viral strains. The broad 
protection conferred by the plHNw-G vaccine confirms these reports and extends them to apply to DNA vaccination.

\section{EFFICACY OF VARIOUS ROUTES OF IMMUNIZATION}

Rainbow trout fry were anaesthetized and immunized with $0.1 \mu \mathrm{g}$ of the plHNVw-G or pLuc DNA vaccines by various routes. Groups of fry were injected with a 30 gauge needle either intramuscularly at the base of the dorsal fin or intraperitoneally at the base of the ventral fins. Fry for the intrabuccal treatment were given the DNA solution by a microtip that was inserted into their throat while the fry were held vertically with the head up. Scarification of the fry skin was performed from the dorsal fin to the abdomen using a 23 gauge needle that contained one drop of DNA vaccine solution $(0.2 \mu \mathrm{g} / \mu \mathrm{l})$ on the bevel. Fry for the gene gun treatment received, on their left lateral side below the dorsal fin, one delivery (325 psi) of $500 \mu \mathrm{g}$ of gold microcarriers ( $1 \mu \mathrm{m}$ in diameter) coated with approximately $0.1 \mu \mathrm{g}$ of DNA. Twenty-nine days post-vaccination, triplicate groups of 15 fish (mean weight, $1.9 \mathrm{~g}$ ) representing each treatment were challenged with IHNV strain $220-90$ at $10^{4}$ plaque forming units (pfu)/ml using standard methods. Vaccine efficacy was determined by comparing the cumulative mortalities and survival times among treatment groups. Fry immunized with the pIHNVw-G DNA vaccine delivered via intramuscular injection, gene gun and intraperitonal injection exhibited significantly $(0<0.01)$ less mortality than fry immunized with the pluc control vaccine. In contrast, groups of fry immunized via scarification of skin and intrabuccal administration did not show significant differences between the pIHNVW-G and pluc vaccines. ${ }^{10)}$

It is well known that the route of delivery of a DNA vaccine in mammals can effect the types and location of cells transfected as well as the nature of the immune response. Previous studies on the mechanisms of action of DNA vaccines in mammals have indicated that only professional antigen presenting cells (APC) are capable of priming immune responses. Somatic tissues that express protein from injected plasmids serve as a reservoir for antigens which are then transferred to professional APCs which use the MHC. presentation pathway and all necessary signals for priming the T-cells. In this study, rainbow trout fry vaccinated via intramuscular injection, or intradermally using the gene gun, were significantly protected against live viral challenge. It is not known if the immune mechanisms in fish are identical upon DNA vaccination into the muscle or skin. However, studies have shown that, similar to mammals, fish antigens are processed and presented by APC such as monocytes and macrophages to specific lymphocytes in a MHClike fashion.

\section{ONSET AND SPECIFICITY OF PROTECTION}

Rainbow trout fry (mean weight, $2 \mathrm{~g}$ ), in groups of approximately 400 fish each, were injected intramuscularty with $1 \mathrm{\mu g}$ of the IHNV DNA vaccine (plHNw-G), or treated as various controls. In the first experiment duplicate groups of 25 fish were removed at $1,7,14,21$, and 28 days postvaccination and challenged by immersion in water containing IHNV at a concentration of $10^{4} \mathrm{pfu} / \mathrm{ml}$. All treatment groups that were challenged 10 after vaccination had high mortality ranging from 61 $86 \%$. In fish challenged at 7 days post-vaccination the plHNw-G groups showed $2 \%$ average cumulative mortality compared with $50-58 \%$ mortality in the various control groups. Similar significant $(p<0.05)$ protection in the plHNw-G vaccinated groups was observed in fish challenged at 14,21 , and 28 days postimmunization. In a second experiment, time points shorter than one week were tested. Rainbow trout fry were vaccinated with the same treatments and controls as above and challenged at $1,2,4$, and 7 days post-vaccination. A higher IHNV challenge concentration of $10^{5}$ pfu/ml was used to provide higher levels of mortality in the control treatments, as a more stringent test of the protection observed. No protection was observed at 1 or 2 days postvaccination, but by 4 days fish immunized with plHNw-G were significantly $(p<0.05)$ protected relative to all controls. By 7 days protection was even stronger, with an average mortality of $20 \%$ in plHNw-G vaccinates compared to $85-94 \%$ in control groups. ${ }^{11)}$

The specificity of this early immunity was investigated using DNA vaccines containing the $G$ genes of two other mabdovirus species. The vaccine denoted here as PVHS-G protects trout against a major European pathogen, viral hemorrhagic septicemia virus (VHSV). VHSV is in 
the same genus as IHNV, but experiments with trout sera show no serological cross-neutralization activity between these viruses. A DNA vaccine against the CVS strain of rabies virus, denoted here as pRV-G, was included as a vaccine for a mammalian rhabdovirus from a different genus. Rainbow trout fry were injected intramuscularly with $1 \mathrm{\mu g}$ of plHNw-G, pVHS-G, pRV-G, or pCDNA3.1 vector alone, or left unhandled. At 4, 7 , 14 , and 28 days post-vaccination duplicate groups of 25 fish were removed from each treatment and challenged by immersion in $10^{4} \mathrm{pfu} / \mathrm{ml}$ of IHNV. The results indicated that significant crossprotection against IHNV challenge could be obtained with the VHSV $G$ gene vaccine for a transient time period. Groups of fish vaccinated with the IHNV DNA vaccine showed significant protection $(p<0.05)$ at all times points, with average cumulative control groups injected with vector alone or left unhandled. Fish vaccinated with the VHSV DNA vaccine showed significant protection ( $p<0.05$ ) ranging 6-20\% CPM at 4,7 , and 14 days post-vaccination, but at 28 days the average mortality of $69 \%$ was as high as in the unhandled control groups. This loss of crossprotection by the VHSV vaccine at later time points was observed in two independent experiments. Fish vaccinated with the rabies DNA vaccine had mortalities comparable to negative control fish at all time points. ${ }^{11)}$

The observation of the onset of protection against IHNV $4 \mathrm{~d}$ after DNA vaccination is the earliest protection time point thus far reported for DNA vaccines. Similarly, Lorenzen et al ${ }^{12)}$ recently showed protection against VHSV 4 days after vaccination of rainbow trout fry with a DNA vaccine containing the $G$ gene of VHSV or IHNV, suggesting that early and semi- specific protection may be a common feature of DNA vaccines against fish habdoviruses. It is also possible that early and semi-specific protection may occur with DNA vaccination in mammalian hosts, but studies describing these features are lacking, likely due to difficulties with using the large numbers of animals needed to test multiple time points and vaccines.

The results of these studies indicated a difference between the mechanism of protection acting at early and later time points postvaccination. It was hypothesized that at least two temporally distinct protective mechanisms exist and that they are initiated by DNA vaccination of rainbow trout. At time points beginning 4 days after vaccination there is an early protective mechanism(s) characterized by rapid onset and low specificity. The consistent protection after $4 \mathrm{~d}$ and the cross-protection results suggest that this mechanism functions for at least 14 days. By 3-4 weeks post-vaccination protection is likely mediated at least to a large extent by more specific adaptive immune response components presumably including both antibodies and cellular factors. It has been shown that the neutralizing antibody response to either virus infection or DNA vaccination in trout is highly specific, with no cross-neutralizing activity between sera from fish infected with IHNV and VHSV or injected with IHNV and VHSV G gene DNA vaccines. It is possible that the loss of the cross-protection against IHNV challenge at the $\mathbf{2 8}$ day time point reflects the down-regulation of the non-specific early protection mechanism when the more specific humoral response becomes active.

\section{CONCLUSION}

Advantages of DNA vaccines over other types of vaccines have been well described. The results described above illustrate the potency of a DNA vaccine against IHNV in rainbow trout and the early and semi-specific protection that are observed in these animals. Different features associated with protection after DNA vaccination could be exploited as effective management tool(s) in a variety of domesticated animal industries including aquaculture. Additionally, these results substantiate that rainbow trout provide an excellent model for understanding host-pathogen interactions and the effect of vaccines in a controlled laboratory setting. Thus, DNA vaccines against IHNV have great potential both as a practical biologic for protection of fish and as an important tool for investigation of the teleost immune system.

\section{ACKNOWLEDGEMENTS}

The authors wish to thank Serge Corbeil from the CSIRO Autralian Animal Health Laboratory, Jerry Jones and Bill Shewmaker from Clear Springs Foods, Ellen Lorenzen and Katja Einer-Jensen from the Danish Veterinary Laboratory , and Eric Anderson from the University of Maine for their significant contributions to these studies. 


\section{REFERENCES}

1. Wolf K. Infectious hematopoietic necrosis. In: Fish Viruses and Fish Viral Diseases. thaca: Cornell University Press, 1988. p 83-114.

2. LaPatra SE. Factors affecting pathogenicity of infection hematopoietic necrosis virus (IHNV) for salmonid fish. $J$ Aquat Animal Health 1998; 10:121-131.

3. Anderson ED, Mourich DV, Fahrenkrug SC, LaPatra SE, Shepherd J, Leong JC. Genetic immunization of rainbow trout (Oncorhynchus mykiss) against infectious hematopoietic necrosis virus. Mol Mar Biol Biotechnol 1996;5:114-122.

4. Corbeil S, LaPatra SE, Anderson ED, Jones J, Vincent $B$, Hus U-L, Kurath $G$. Evaluation of the protective immunogencity of the N,P,M,NV and $G$ proteins of infectious hematopoietic necrosis virus in rainbow trout (Oncorhynchus mykiss) using DNA vaccines. Dis Aquat Org 1999;39:29-36.

5. Traxler GS, Anderson E, LaPatra SE, Richard J, Shewmaker WS, Kurath $G$. Naked DNA vaccination of Atlantic salmon (Salmo salar) against IHNV. Dis Aquat Org 1999;38:183-190.

6. LaPatra SE, Turner T, Lauda KA, Jones GR, Walker $\mathrm{S}$. Characterization of the humoral response of rainbow trout to infectious hematopoietic necrosis virus. J Aquat Anim Heath 1993;5:165-171.
7. Saporito-Irwin SM, Geist RT, Gutmann DH. Ammonium acetate protocol for the preparation of plasmid DNA suitable for mammalian cell transfections. Blotechniques 1997;23:424-427.

8. Corbeil S, LaPatra SE, Anderson ED, Kurath G. Nanogram quantities of a DNA vaccine protect rainbow trout fry against heterologous virus strains of infectious hematopoietic necrosis virus. Vaccine 2000;18:2817-2824.

9. LaPatra SE, Corbeil S, Jones GR, Shewmaker WD, and Kurath $G$. The dose-dependent effect on protection and humoral response to a DNA vaccine against IHN virus in subyearling rainbow trout. $J$ Aquat Anim Health 2000;12:181-188.

10. Corbeil S, Kurath G, LaPatra SE. Fish DNA vaccine against infectious hematopoietic necrosis virus: efficacy of various routes of immunization. Fish Shelfish Immunol 2000; 10:711-723.

11. LaPatra SE, Corbeil S, Jones GR, Shewmaker WD, Lorenzen N, Anderson ED, Kurath $G$. Four days after DNA vaccination rainbow trout exhibit significant, semi-specific protection against lethal virus challenge in rainbow trout. Vaccine 2001;19:4011-4019.

12. Lorenzen N, Lorenzen E, Einer-Jensen K, LaPatra SE. Immunity induced shortly after DNA vaccination of rainbow trout against mabdoviruses protects against heterologous virus but not against bacterial pathogens. Dev Comp Immunol ;in press. 